\title{
Philosophiques
}

\section{Spinoza et Darwin, un héritage véritablement conceptuel ?}

\section{Vincent Legeay}

Volume 45, numéro 2, automne 2018

URI : https://id.erudit.org/iderudit/1055271ar

DOI : https://doi.org/10.7202/1055271ar

Aller au sommaire du numéro

Éditeur(s)

Société de philosophie du Québec

ISSN

0316-2923 (imprimé)

1492-1391 (numérique)

Découvrir la revue

Citer cet article

Legeay, V. (2018). Spinoza et Darwin, un héritage véritablement conceptuel ? Philosophiques, 45(2), 445-459. https://doi.org/10.7202/1055271ar

\section{Résumé de l'article}

L'article entend proposer un critère d'évaluation d'un héritage conceptuel possible de Spinoza à Darwin, mettant en avant qu'il ne suffit pas de mobiliser des notions communes entre les deux auteurs. Pour ce faire, il commence par restituer une série de comparaisons contemporaines dans cette voie, pour ensuite tenter d'expliciter ce que Darwin entend lui-même demander à la mobilisation de certaines de ses notions héritées. C'est alors sur le critère de la différence individuelle que l'analyse permettra de dégager un schéma conceptuel suffisamment probant pour dire que Darwin reprend une pensée historiquement située. Enfin, après avoir étudié une divergence conceptuelle significative dans cette reprise, l'analyse montre que la voie darwinienne laisse délibérément de côté une dimension ouverte de l'analyse spinoziste concernant le concept de supériorité (praestantia). 


\title{
Spinoza et Darwin, un héritage véritablement conceptuel?
}

\author{
VINCENT LEGEAY \\ Université Paris 1 \\ Vincent.legeay@gmail.com
}

\begin{abstract}
RÉSUMÉ. - L'article entend proposer un critère d'évaluation d'un héritage conceptuel possible de Spinoza à Darwin, mettant en avant qu'il ne suffit pas de mobiliser des notions communes entre les deux auteurs. Pour ce faire, il commence par restituer une série de comparaisons contemporaines dans cette voie, pour ensuite tenter d'expliciter ce que Darwin entend lui-même demander à la mobilisation de certaines de ses notions héritées. C'est alors sur le critère de la différence individuelle que l'analyse permettra de dégager un schéma conceptuel suffisamment probant pour dire que Darwin reprend une pensée historiquement située. Enfin, après avoir étudié une divergence conceptuelle significative dans cette reprise, l'analyse montre que la voie darwinienne laisse délibérément de côté une dimension ouverte de l'analyse spinoziste concernant le concept de supériorité (praestantia).
\end{abstract}

\begin{abstract}
A mere succession of notions through time is ineffective in order to show a real and deep theoretical relationship between two authors of two different times. This article is designed to provide a strong evidence of a conceptual inheritance from Spinoza to Darwin. It starts with a comparison between different declarations of a contemporary author who have lately made a similar attempt, and follows by a forward analysis of Darwin's own views and declarations concerning his using of several notions close to those of Spinoza. It is therefore advocated that it is mainly the concept of difference that ensures an authentic inheritance of a conceptual scheme between the dutch and english thinkers. After proving that Darwin is accurately aware of that historical theoretical framework, the analysis will eventually consider that the naturalist left aside one of Spinoza's biological outbreaks concerning the notion of the individual superiority (what Spinoza used to call " praestantia ").
\end{abstract}

Dans son ouvrage récent, Café Spinoza ${ }^{1}$, Michel Juffé opère au chapitre III un rapprochement entre les deux auteurs à partir de plusieurs textes; dont celui du chapitre VII de la quatrième partie de l'Éthique, dans sa propre traduction:

Il n'est pas possible que l'homme ne soit pas une partie de la Nature et qu'il n'en suive pas l'ordre commun. Pourtant s'il vit parmi des individus dont la nature s'accorde avec la sienne, sa puissance d'agir sera par là même secondée et favorisée; s'il vit au contraire parmi des individus qui ne s'accordent que

1. Éditions Le Bord de l'Eau, 2017.

PHILOSOPHIQUES 45/2 - Automne 2018, p. 445-459 
fort peu avec sa nature, il ne peut guère s'adapter à eux sans opérer en luimême une grande mutation ${ }^{2}$.

Après avoir précisé qu'aucun individu «ne peut prétendre assurer une supériorité sur les autres ", M. Juffé glose l'utilisation du terme de "mutation " en prévenant toute anticipation spinoziste de la génétique. Il propose de considérer le parallèle entre les deux auteurs en mobilisant un autre texte du chapitre III de L'origine des espèces:

Il ne faut jamais oublier que chaque être organisé s'efforce toujours de se propager [...] Tous les êtres organisés s'efforcent perpétuellement de se multiplier selon une progression géométrique.

Le sens donné par le néerlandais au terme mutatio ne peut absolument pas, de pleine rigueur, être envisagé selon l'angle darwinien, ni selon l'angle biologique contemporain de la théorie synthétique de l'évolution. Il possède le sens tout à fait cartésien du mouvement, donc d'un changement par déplacement mécanique. De même, la traduction du verbe «accommodare » telle qu'elle est présentée par Michel Juffé, pose problème. Le terme d'adaptation semble renvoyer de façon faussement évidente à la fois au langage spinoziste et au système darwinien, alors que les termes propres à chacun de ces auteurs ne sont pas identiques. Dans le cas de Spinoza, il s'agit de l'accommodement, dans celui de Darwin de l'adaptation.

Est alors mobilisée une comparaison de l'utilisation commune de la lutte pour la persistance et de l'accumulation de variations ou mutations expliquant l'émergence continue d'individualités complexes (au double sens de leur composition organique et du type d'analyse qu'il est nécessaire de mobiliser pour expliquer les caractéristiques de ces individus).

L'auteur mobilise donc un ensemble de «notions» et d'entrées lexicales pour exposer le rapprochement possible entre les pensées de Spinoza et de Darwin. La comparaison n'est pourtant pas évidente. D’abord parce que Spinoza ne rechigne pas à utiliser certaines autres notions, comme celle de "forme ", d' " espèce " ou d' " essence » dans l'ensemble de son corpus théorique, et à en faire un usage qui peut sembler globalement classique du point de vue des écoles métaphysiques traditionnelles (scolastique ou cartésienne) à son époque ${ }^{3}$. Ensuite parce qu'il ne peut exister chez ce dernier ni de

2. Le terme latin est en effet «magna mutatio». Précisons toutefois que la traduction de Pautrat, qui constituera pour nous la référence dans cet article (sauf mention contraire), donne «grand changement». De même, la traduction du verbe "accommodare» pose un problème. Pour un plaidoyer en faveur du maintien en français du verbe "s'accommoder ", voir l'article d'Ariel Suhamy dans l'ouvrage L'essence plastique, Vincent Legeay (dir.), Éditions de la Sorbonne, Paris, 20I8, auquel nous nous permettons de renvoyer.

3. Pour une autre référence plus juste, mais seulement allusive, rapprochant l'antifinalisme et l'anti-essentialisme classique de Spinoza à l' "évolutionnisme » darwinien, on lira le livre de Daniel Dennett, Darwin est-il dangereux?, Odile Jacob, collection «Philosophie", Paris, 2000. 
théorie de la sélection calquée sur celle de l'état domestique, ni encore de théorie de l'hérédité génétique (que ce soit par mutation ou par transformation). Enfin parce que la théorie darwinienne repose en grande partie sur un système scientifique considérablement développé, dont les deux disciplines de base sont l'embryologie et la paléontologie, possédant un stock de connaissances en I 859 littéralement incommensurable à celui de la moitié du XVII siècle.

Cependant, il est tout à fait certain que sur quelques "éléments théoriques " bien particuliers, il existe une reprise des thèmes spinozistes. Allons plus loin: il existe, selon nous, un héritage solide de certains concepts spinozistes, tout à fait évident, et même d'un appareil conceptuel spinoziste qui doit être précisé comme tel. Or, précisément, il s'agit d'un héritage bien précis et particulier, qui doit être restitué entièrement, sans que l'on puisse véritablement dire que Spinoza soit à ce compte un "précurseur ${ }^{4}$. La preuve d'une conceptualité héritée ne peut se faire par la simple superposition de termes (tous susceptibles de changer de sens, donc également de référents). Il est a minima désirable de mobiliser un - voire plusieurs intermédiaire théorique, historique, philologique. Inversement, cet héritage ne doit pas lui-même être renversé sous la forme d'une pré-conceptualité déjà à l'œuvre avant même l'utilisation selon les nouveaux cadres scientifiques du même vocabulaire. Il existe une différence entre héritage lexical et héritage conceptuel qui doit être questionnés.

Notre question est donc simple: y a-t-il un sens à opérer une comparaison entre certains concepts spinozistes et darwiniens qui ne soit pas seulement la superposition de termes ou de notions se ressemblant? Si oui, quel intermédiaire théorique pourrait justifier l'idée d'un héritage conceptuel entre les deux auteurs? À cette double question, nous attachons un enjeu bien précis, qui est celui de comprendre en quoi la pensée spinoziste pourrait permettre de comprendre une certaine orientation de la pensée darwinienne, pour en mesurer à la fois la profondeur et la relativité historique. C'est cette inflexion dans l'histoire de la pensée, précession, plutôt que la mobilisation d'éléments lexicaux de prédécesseur que nous voulons analyser ici.

\section{Trouver un héritage certain}

Au-delà des filiations lâches, longues, mais certaines, qui unissent la pensée de Spinoza à celle de la variation, antérieurement à tout le fil du matérialisme issu de la pensée lucrécienne, et prospectivement à l'héritage d'un matérialisme aléatoire que récupérera Althusser, un triple indice textuel

4. Le terme apparaît cependant dans le livre récent de Michel Juffé, qui nomme Spinoza, page 43, "un des précurseurs » de l'écologie telle que Darwin a permis de la penser.

5. Pour une présentation des complexités à établir des interprétations d'héritage, on lira le dernier ouvrage de Chantal Jaquet sur la question Spinoza à l'œuvre. Composition des corps et force des idées, Éditions de la Sorbonne, collection «La philosophie à l'œuvre ", Paris, 2017. 
nous donne immédiatement la mesure de l' "espoir théorique » que constitue tout rapprochement entre Spinoza et la future pensée darwinienne.

D'abord, l'apparition dans le titre même du chapitre IV, de l'idée de "fittest», traduite en français très justement par "plus apte». Immédiatement un double enthousiasme saisit un lecteur spinoziste, suivi d'une grande prudence. Enthousiasme parce que c'est à la notion de "persistance» que l'" aptitude» est accolée, et que les deux notions sont des pivots de la "physique spinoziste». Enthousiasme aussi parce que l'histoire philosophique anglaise elle-même du terme de fitness doit être retracée à des auteurs proches de Spinoza, comme Hobbes par exemple, et qu'une histoire de cette conceptualité est permise grâce à des transfuges théoriques tout à fait reconnaissables dès l'époque de Spinoza. Mais grande prudence également parce que, comme explicitement mentionné, cette expression est celle d'Herbert Spencer, dont la marque propre pose des difficultés théoriques et historiques en regard de la compréhension de Darwin lui-même $e^{6}$. Même si nous laissons ces difficultés de côté ici, il reste problématique de lier la pensée spinoziste et la pensée darwinienne à travers la convocation de termes émanant d'un autre auteur. C'est pourquoi il nous faut dès maintenant considérer les conditions d'une appropriation de ces concepts par Darwin seul.

Et justement, la seconde indication textuelle est fournie par Darwin lui-même au chapitre III de L'origine des espèces, qui explique que la formule de Spencer est tout à fait précise et juste. Dans la dernière édition, celle de 1872 , il déclare en effet:

J'ai donné à ce principe, en vertu duquel une variation si insignifiante qu'elle soit se conserve et se perpétue, si elle est utile, le nom de sélection naturelle, pour indiquer les rapports de cette sélection avec celle que l'homme peut accomplir. Mais l'expression qu'emploie souvent M. Herbert Spencer: «la persistance du plus apte", est plus exacte et quelquefois tout aussi commode [But the expression often used by Mr. Herbert Spencer, of the Survival of the Fittest, is more accurate, and is sometimes equally convenient.]. Nous avons vu que, grâce à la sélection, l'homme peut certainement obtenir de grands résultats et adapter les êtres organisés à ses besoins, en accumulant les variations légères, mais utiles, qui lui sont fournies par la nature.

On le voit, Darwin ne fait pas que récupérer l'expression d'un confrère, il la commente comme plus exacte et aussi commode que la sienne. Remarquons également qu'elle confère un statut directement explicatif à la complexité individuelle, puisque la persistance (survival) n'est garantie que par un ensemble de variations légères et utiles accumulées et nommées «fit» ou «apte» en français. Il existe donc une forme de raison à motiver les preuves d'un rapprochement Spinoza/Darwin sur le caractère à la fois individuel et

6. Pour un éclaircissement des rapports extrêmement intriqués et difficiles de Darwin et de ses contemporains, on lira l'ouvrage de Jean Gayon Darwin et l'après-Darwin. Une histoire de l'hypothèse de sélection naturelle, Éditions Kimé, "Histoire des idées", Paris, I992. 
complexe de la variation, puisque la sélection naturelle darwinienne, tout comme l'accommodatio et l'aptitudo spinozistes, n'agissent que par l'accumulation de variations individuelles. C'est aussi que, dans chacun des cas, l'individu est saisi par le type de relations qu'il entretient avec l'ensemble des autres individus, qu'ils soient biotiques ou abiotiques. Chez Darwin, la relation est directement compétitive et trophique, l'individu n'étant qu'un résidu de la structure d'interactions; chez Spinoza, la relation est également oppositive et trophique, l'aptus étant lié à l'alimentation.

On remarque dans la fin du passage sus-cité également la très grande proximité de la remarque sur l'adaptation des êtres organisés aux besoins humains avec le chapitre XXVI de l'appendice de la quatrième partie de l'Éthique, où Spinoza déclare à propos des êtres organisés qui ne sont pas des hommes:

Elle [la règle de notre utilité] nous enseigne, en vue d'usage divers, à le conserver, à le détruire ou à l'adapter [conservare, destruere vel adaptare] à notre usage de quelque façon que ce soit.

Seulement, Darwin reprend cette idée d'une manière indéniablement transposée ${ }^{7}$ : l'adaptation ne vaut comme concept que parce qu'il est hérité d'une compréhension fortement humaine de l'usage conforme des choses extérieures à certaines finalités favorables. C'est alors au moyen de cette transposition que l'adaptation peut être comprise comme un concept darwinien hérité de Spinoza; à la prudence près que Spinoza ne l'utilise pas ainsi, mais privilégie le terme d'accommodare pour rendre compte de l'accumulation de petites variations et changements expliquant l'aptitude individuelle.

La dernière indication textuelle, qui concorde avec cette interprétation, tout en paraissant être un renoncement, est livrée dans une lettre du 5 juillet I 866 de Darwin à Wallace, où le premier commente encore l'expression de Spencer:

Je tombe d'accord avec vous sur tous les avantages que vous nommez dans l'utilisation de l'excellente expression de H. Spencer «la persistance des plus aptes». [...] Cependant, le fait que ce terme ne puisse être utilisé comme substantif commandant un verbe est une grande objection contre lui; objection réelle que je déduis de $\mathrm{H}$. Spencer lui-même puisqu'il utilise continuellement les mots de sélection naturelle. [I fully agree with all that you say on the advantages of H. Spencer's excellent expression of "the survival of the fittest".

7. Nous nous opposons donc au rapprochement établi par M. Juffé dans la section de son livre, op.cit, qui concerne le fait de traiter les bêtes à notre convenance. Ce n'est pas directement qu'un héritage conceptuel entre Darwin et Spinoza peut être établi, mais de façon transposée, précisément parce que Darwin pense la sélection naturelle comme une généralisation du principe de sélection domestique, comme il l'établit dans le premier chapitre de L'origine des espèces. Le verbe spinoziste adaptare qui signifie le maniement des choses extérieures pour conformer des individus à nos fins propres, prend donc sens dans la théorie darwinienne au prix d'une transposition, ce qui est très important. 
[...] It is, however, a great objection to this term that it cannot be used as a substantive governing a verb; $\sigma$ that this is a real objection I infer from $\mathrm{H}$. Spencer continually using the words natural selection $]^{8}$.

Darwin plaide pour l'emploi d'un substantif. À première vue, c'est bien ce que représenterait la notion de «survival» en anglais. Que veut-il donc dire? Hors la contradiction, la seule possibilité est qu'il se réfère au terme «fit». Immédiatement, pourtant, Darwin pourrait proposer d'utiliser le terme de "fitness». Il n'ignore pas le terme puisqu'il l'utilise à deux reprises dans le corps du texte de son opus major' ${ }^{9}$. Pourquoi une méfiance vis-à-vis de ce terme? Peut-être d'abord parce qu'en I 866 Darwin n'est pas encore prêt, comme il le sera six ans plus tard, à accorder entièrement la validité de l'expression, qui est une concession faite à un collègue. Ensuite, peut-être parce que ce terme de fitness possède déjà une histoire marquée, comme moyen de "distinction" des individus à la fois physique et social ${ }^{10}$. Cette idée nous semble une hypothèse d'autant plus importante que certains sectateurs de Darwin reprendront directement le terme, propagé par Spencer. Darwin, qui n'utilise l'adaptation que de façon transposée, semble hésiter à reprendre un vocabulaire non plus seulement de sélection domestique, mais

8. Notre traduction. Idem pour les futures références citées.

9. Chaque fois de façon soit négative soit distanciée, cependant. La première occurrence se trouve dans le chapitre VII concernant les objections contre la sélection naturelle, la seconde dans le dernier chapitre au moment de considérer certaines «erreurs » de la nature qui serviraient d'entraves à la puissance explicative de la sélection naturelle.

10. Hobbes, dans le Leviathan, distingue entre une capacité de simple accès à des images et des affections différentes, et la faculté d'opérer un tri parmi celles-ci, toutes deux classées sous le genre unique de l' «ability» ou du «wit» (ou encore «witte»), vertu intellectuelle qui peut elle-même être redistribuée selon deux sous-espèces. Hobbes marque une double différence entre ces vertus en distinguant entre les vertus naturelles et les vertus acquises, ce qui place l'ability directement dans une dimension biologique prononcée, puisqu'elle suit les mouvements plus ou moins rapides de l'imagination, entretenus par l'usage (use) et l'expérience. L'auteur précise bien que cette ability, est discrétionnaire tout autant que commune: les hommes la désirent communément pour eux-mêmes, mais elle permet également de faire des différences entre les individus ( the same word Witte, be used also, to distinguish one certain ability from the rest»). Or la distinction opérée par Hobbes dans le chapitre VIII est intéressante dans la mesure où l'" habileté naturelle" elle-même est scindée d'une double façon. La première ability est celle d'enchaîner de façon plus ou moins rapide donc plus ou moins redondante des images en n'apercevant entre elles que de simples similitudes, alors que la seconde établit des différences entre ces affections-perceptions. Dès lors l'ability ou le wit est dite soit tout simplement une «bonne imagination» («a good fancy») soit un bon «discernement» ( discretion»).

Certes, ces abilities ne sont pas nommées des «aptitudes ", bien qu'elles s'en rapprochent fortement. À l'appui de cette compréhension corporelle des enchaînements intellectuels, Hobbes va proposer plus loin, dans le chapitre X, le terme d' "aptitude» (dont l'édition anglaise précise "fitness, or aptitude" et la latine "dignitas pro aptitudine»), dont la première occurrence côtoie la notion de "compétence» ("worthiness" en anglais), à teneur très sociale. Il reprend ici, précisément, le caractère discrétionnaire de l'ability développé au chapitre VIII. 
de discrimination sociale, afin de nommer toute individualité persistée selon l'accumulation complexe de variations.

Car Darwin est bien à la recherche d'une forme de distinction des individualités afin de rendre compte des variétés devenant des espèces. Reprenons le texte du troisième chapitre, légèrement étendu:

On peut encore se demander comment il se fait que les variétés que j'ai appelées espèces naissantes ont fini par se convertir en espèces vraies et distinctes, lesquelles, dans la plupart des cas, diffèrent évidemment beaucoup plus les unes des autres que les variétés d'une même espèce; comment se forment ces groupes d'espèces, qui constituent ce qu'on appelle des genres distincts, et qui diffèrent plus les uns des autres que les espèces du même genre? Tous ces effets, comme nous l'expliquerons de façon plus détaillée dans le chapitre suivant, découlent d'une même cause: la lutte pour l'existence. Grâce à cette lutte, les variations, quelque faibles qu'elles soient et de quelque cause qu'elles proviennent, tendent à préserver les individus d'une espèce et se transmettent ordinairement à leur descendance, pourvu qu'elles soient utiles à ces individus dans leurs rapports infiniment complexes avec les autres êtres organisés et avec les conditions physiques de la vie.

Le projet darwinien est essentiellement une tentative de cerner au niveau des singularités les critères d'une distinction biologique permettant d'observer l'apparition d'une espèce différente. Que cette distinction soit appelée «sélection naturelle» ou "persistance des plus aptes", ou fitness, n'est en soi qu'un indice d'une reprise d'un héritage plus profond: Darwin établit une liaison d'une part entre le fait d'être apte ( fit) et l'observation d'une distinction individuelle dont il semble apercevoir la dimension socialement importée, d'autre part entre cette distinction et l'accumulation de variations. Or c'est ce double mouvement qui qualifie très rigoureusement la conception de l'aptus spinoziste (dont, notons-le immédiatement, Spinoza ne fit jamais un substantif). Cette conception est elle-même importée d'une compréhension hobbesienne de la fitness en tant qu'élément discriminatoire pour ce qui distingue (differ en anglais, et praestat en latin) un individu d'un autre, au sein de l'espèce humaine, voire entre l'espèce humaine et une autre. C'est dans cette histoire théorique-là, pensons nous, que peut être établie une filiation entre Darwin et Spinoza, plutôt qu'en superposant certains termes qui se «ressemblent», comme celui de mutatio.

\section{Une histoire de différence}

Il est impossible de faire sous format court l'histoire de cette conceptualité partagée par Spinoza et Hobbes, sous-tendant une grande partie des conceptions matérialistes ultérieures concernant les "états naturels». Pour ce qui nous concerne, il suffit d'étudier la façon dont a pu être hérité l'ensemble conceptuel suivant:

(accumulation de variations + sélection des changements utiles) $\times$ contrainte $d u$ contexte $=$ aptitude 
L'aptitude permet alors la reconnaissance qu'une différence singulière est la source d'explication complexe d'individus distincts.

C'est ce groupe conceptuel (les anglo-saxons disent cluster concept) dans sa globalité qui semble être pratiquement identique chez Spinoza et Darwin. Il nous semble alors que la preuve de cet héritage est d'autant plus forte qu'il s'agit d'un "schéma» se retrouvant, efficace, dans l'ouvrage du naturaliste anglais. Remarquons avant toute chose que la notion d' "adaptation» ne rentre pas dans cet héritage, dont nous avons dit qu'elle était reprise sous la forme d'une transposition par Darwin, si l'on désigne par là l'adaptare spinoziste ${ }^{11}$. Cette transposition possède elle-même une histoire qu'il serait trop long de restituer ici.

In fine, c'est le critère de la différence qui provoque l'héritage du groupe conceptuel dans sa globalité, parce qu'elle se situe au départ et au résultat de l'analyse scientifique dans les deux cas. Que voulons-nous dire?

Cette notion de "différence" est doublement opératoire. D'une part, elle est «ce qui apparaît» d'abord au naturaliste, sans qu'il ait pu encore déterminer les changements ayant préparé un avantage individuel. C'est sous l'angle d'une distinction que l'individu est «repéré » par le naturaliste. Mais ce n'est que la première étape. Darwin le dit lui-même dans cette phrase qui précède immédiatement le dernier paragraphe cité:

Le seul fait de l'existence de variabilités individuelles et de quelques variétés bien tranchées, quoique nécessaires comme point de départ pour la formation des espèces, nous aide fort peu à comprendre comment se forment ces espèces à l'état de nature, comment se sont perfectionnées toutes ces admirables adaptations d'une partie de l'organisme dans ses rapports avec une autre partie, ou avec les conditions de la vie, ou bien encore, les rapports d'un être organisé avec un autre.

La différence est efficace à un second niveau, elle rend compte d'une véritable supériorité ${ }^{12}$, lorsque cette première distinction individuelle ren-

11. Par opposition à l'accommodare spinoziste, qui convient parfaitement à la compréhension de contrainte environnante jouant le rôle d'une sollicitation constante des individus devant eux-mêmes sélectionner les affections et changements utiles. En ce sens, nous donnons raison à Ariel Suhamy dans son article déjà cité, lorsqu'il propose de maintenir une traduction littérale de l'accommodatio. Qu'il n'existe pas de théorie de l'adaptation chez Spinoza n'empêche pas que l'aptitude soit un produit individuel de l'accommodement général.

12. Concept rejeté par Michel Juffé pour décrire les individus humains, alors que la notion est omniprésente dans le texte darwinien. Que les individus humains ne soient pas une exception dans la nature est tout-à-fait juste, mais qu'ils soient supérieurs à beaucoup d'autres individus semble indéniable, pour peu qu'on entende par là ce que Spinoza comme Darwin pensent de cette supériorité, concept absolument neutre et désignant l'ensemble des variations favorables. Juffé semble nier ce concept sur une base prudente de soupçon d'anthopocentisme. La notion de praestantia spinoziste ou de superiority darwinienne dénotent pourtant seulement une situation où la distinction est favorable, donc aide à la conservation des individus. Il faut la trichotomie conceptuelle pour penser la supériorité. Un exemple parmi beaucoup d'autres occurrences, dans le chapitre IV de L'origine des espèces: "We have seen that it is the common, 
contre fonctionnellement la contrainte exercée par les conditions extérieures et que les changements produits commencent à être triés en fonction de leur utilité. Darwin explique, dans un passage du chapitre IV:

Je comprenais l'immense importance des différences individuelles, et cela m'avait conduit à discuter en détail les résultats de la sélection inconsciente par l'homme, sélection qui dépend de la conservation de tous les individus plus ou moins supérieurs et de la destruction des individus inférieurs.

La transposition domestique/naturelle joue de nouveau ici, mais elle n'est que secondaire vis-à-vis de ce concept premier des différences individuelles. Le naturaliste cherche avant tout à rendre explicable une distinction empirique - seulement constatée - au moyen d'une différence favorable, fonction des circonstances (elles-mêmes comprises par l'accumulation des preuves fossiles). Or, aussi surprenant que cela puisse paraître, cette compréhension darwinienne de l'adaptation individuelle liant différence supériorité - distinctionlaptitude est l'exact équivalent, dossiers fossiles en plus, de la compréhension spinoziste. Nous ne citerons ici que deux textes du philosophe néerlandais, mis en regard. Le premier prépare la "petite physique ", et ancre le programme de l'aptitude dans une étude des supériorités individuelles:

Mais cette nature, je ne peux l'expliquer ici, et cela n'est pas nécessaire pour ce que je veux démontrer. Je dis pourtant, de manière générale [in genere tamen], que plus un Corps l'emporte sur les autres [Corpus aliquod reliquis aptius] par son aptitude à agir et pâtir de plus de manières à la fois [ad plura simul agendum, vel patiendum], plus son Esprit l'emporte sur les autres par son aptitude à percevoir plus de choses à la fois [ad plura aptior simul percipiendum]; et plus les actions d'un corps dépendent de lui seul et moins il y a d'autres corps qui concourent avec lui pour agir, plus son esprit est apte à comprendre de manière distincte [aptior ad distinctè intelligendum]. Et c'est par là que nous pouvons connaître la supériorité d'un esprit sur les autres [cognoscere possumus praestantiam unius mentis].

the widely diffused, and widely ranging species, belonging to the larger genera within each class, which vary most; and these tend to transmit to their modified offspring that superiority which now makes them dominant in their own countries. Natural selection, as has just been remarked, leads to divergence of character and to much extinction of the less improved and intermediate forms of life. On these principles, the nature of the affinities, and the generally well defined distinctions between the innumerable organic beings in each class throughout the world, may be explained. It is a truly wonderful fact - the wonder of which we are apt to overlook from familiarity - that all animals and all plants throughout all time and space should be related to each other in groups, subordinate to groups, in the manner which we everywhere behold - namely, varieties of the same species most closely related, species of the same genus less closely and unequally related, forming sections and sub-genera, species of distinct genera much less closely related, and genera related in different degrees, forming subfamilies, families, orders, sub-classes, and classes." 
Rappelons immédiatement que l'esprit chez Spinoza désigne la même chose que le corps, conçu sous un attribut différent. Le concept de distinction chez l'amstellodamois agit doublement: il explique comment l'individu se distingue au sens où il accumule un nombre croissant de variations utiles corporelles et mentales (l'ensemble des occurrences de l'aptitude dans le corps de l'Éthique est articulé à cette notion d'utilité), distinction toute intérieure, qui trie pour soi les affections utiles. Distinction inter-individuelle également, exprimant un rapport de comparaison lié à l'ensemble des autres individus, qui sont des parties de la nature au même titre. Spinoza précise, dans le chapitre VI de l'appendice de la quatrième partie, le paramètre effectif qui permet alors d'organiser cette sélection des affects utiles:

Mais comme ce dont l'homme est cause efficiente est nécessairement bon, il ne peut donc rien arriver de mauvais à l'homme sinon par des causes extérieures; à savoir, en tant qu'il est une partie de la nature tout entière, aux lois de laquelle la nature humaine est forcée d'obéir, et à laquelle elle est forcée de s'adapter d'une infinité presque de manières [nempe quatenus pars est totius naturae, cujus legibus humana natura obtemperare et cui infinitis modis penè sese accommodare cogitur $]^{13}$.

La contrainte naturelle agit sur les individus d'une infinité presque de manières, ce qui pose clairement le type de fonctionnalité naturelle rendue effective par l'accumulation de variations des parties individuelles. L'individu «obéit» (paret) à la nature, et même, comme nous avons vu plus haut, "autant que la nature des choses l'exige» (exigit). La marque distincte des individus aptes, c'est de répondre à un ensemble quasi-infini de mutationes par une forme d'indépendance affective, que Spinoza nomme praestantia.

Assurément, le concept de praestantia est traduit partout, dans toutes les traductions de l'Éthique, par le substantif «supériorité ». Il doit pourtant être compris, pensons-nous, comme un simple marqueur de la complexité d'un individu ayant réussi à organiser favorablement ses changements intérieurs - en nombre fini - en fonction de cette contrainte quasi-infinie. Il faut le comprendre donc comme une prae-stantia: l'aptitude à se tenir fonctionnel prioritairement et face aux circum-stantiae. En ce sens, il s'agit bien d'un concept neutre de distinction individuelle réussie, comme l'est aussi celui tout-à-fait neutre de supériorité chez Darwin.

\section{Un point de disjonction?}

Récupérons cet acquis. La différence individuelle constatée, explicable au moyen d'un facteur contraignant, celui des circonstances extérieures, peut devenir une véritable distinction, à savoir une supériorité neutre face au contexte contraignant. Un point de disjonction oppose cependant, sur le pas

13. Le texte latin dit «sese accommodare", Pautrat traduit néanmoins par "s'adapter». Nous avons vu plus haut que cela posait un problème. 
théorique suivant, nos deux auteurs. La différence mène pour Spinoza à un changement de référence de l'individu, qui de "peu apte à connaitre la nature ", devient très conscient de celle-ci, et donc peut être dit en position de prae-stantia parce qu'il fait face à chaque contexte affectif et changeant avec pour connaissance prioritaire le type d'infini naturel qui revient à ce contexte. La différence mène pour Darwin à un changement de catégorisation: de simple variété, l'individu peut devenir une espèce parce que génétiquement devient possible la reproduction de cette variation en survivant. La persistance obtenue dans la différence est transportée sur une échelle phylogénétique chez l'auteur anglais, là où elle était ontogénétique chez le néerlandais. Ainsi, ce à quoi se réfèrent, c'est-à-dire se rapportent les individus, c'est dans le premier cas une Nature infinie simultanée, conçue distinctement parce que non plus indexée sur les circumstantiae fortuites, dans le second cas une histoire naturelle prolongée grâce à une reproduction génétique, et aménagée par la culture.

De même, les auteurs ne se rejoignent pas sur l'instance de la «sélection" des changements (accommodatio pour le premier, adaptation pour le second). En effet, pour Spinoza, c'est l'individu qui «trie» les affections utiles, et sa persistance s'explique avant tout par un grand changement de lui-même, comme le mentionne la proposition 4 de la quatrième partie mentionnée plus haut (expression traduite par "grande mutation" chez Juffé, alors qu'il s'agit en toute rigueur d'un "grand changement", puisque Spinoza désigne par là l'ensemble du rapport de mouvement et de repos entre les parties). Dans le cas de Darwin, c'est la nature, à savoir donc l'ensemble contraignant extérieur - équivalent des circumstantiae - qui sélectionne les changements utiles, et l'individu adapté est moins le produit d'un grand changement de soi-même que le survivant d'une lutte qu'il ne maitrise absolument pas.

Il existe donc une légère différence d'accent entre les deux auteurs: dans le premier cas l'individu en changeant de référence, donc en se différenciant, est le véritable auteur de l'accommodement; dans le second cas, c'est la nature elle-même qui est l'instance différentielle, car c'est d'elle qu'émane la complexité à l'origine de l'accroissement d'une distinction individuelle sous la forme d'une variété. Cette disjonction ne change pas l'héritage conceptuel global que nous avons repéré plus haut, mais elle est importante dans la mesure où elle circonscrit à partir d'une même racine conceptuelle, à même cette racine pourrait-on dire, une première fourche théorique tranchée.

Dans tous les cas, certes, l'individu humain ne fait pas exception à cette règle, mais il produit une supériorité neutre, à savoir une marque distinctive de la complexité développée, afin de se placer en position privilégiée dans la nature. Mais alors, immédiatement, doivent s'imposer et s'appliquer les remarques que nous venons de faire sur les individus humains et le type de «rapports» qu'ils entretiennent avec la Nature. Darwin, dans le même chapitre III, nous donne un bon indice de l'impact réel d'une telle conception: 
Un corollaire de la plus haute importance doit être déduit de ces remarques, à savoir que la structure de chaque être organisé se rapporte, de la façon la plus essentielle, bien que souvent cachée, à celle de tous les autres êtres organisés, avec lesquels il entre en compétition pour sa nourriture et son habitat, qu'il doit fuir parfois, qu'il chasse d'autres fois. [A corollary of the highest importance may be deduced from the foregoing remarks, namely, that the structure of every organic being is related, in the most essential yet often hidden manner, to that of all other organic beings, with which it comes into competition for food or residence, or from which it has to escape, or on which it preys $]^{14}$.

L'application de cette "loi générale» à l'individu humain possède le mérite d'établir une forme de clarté (nous pourrions dire de "distinction" de second degré) immédiate: les humains, aussi complexes soient-ils, aussi distincts soient-ils, c'est-à-dire aussi supérieurs — dans le sens neutre que nous avons dégagé - soient-ils, doivent leur distinction autant à eux-mêmes qu'aux autres êtres organisés, qu'ils soient humains ou animaux. Le changement de référence n'est donc pas un isolement, une mise à l'écart. L'individu distinct ne se rapporte jamais, dans sa manière d'être comme dans la façon dont il s'explique, qu'aux autres individus entretenant avec lui des rapports étroits de contrainte ou de coopération. Qu'il soit en position démarquée ou privilégiée ne change pas la façon dont sa distinction complexe s'explique: par l'ensemble des autres "facteurs biotiques", pour reprendre un terme de l'écologie contemporaine.

Il est possible d'aller plus loin, et - retenant une leçon darwinienne autant que spinoziste, comme nous allons le voir - de mobiliser le critère des facteurs non biotiques pour comprendre ce changement de référence engagé dans chacune des conceptions. Darwin, toujours au même chapitre, avait déjà fait cette déclaration:

Grâce à cette lutte, les variations, quelque faibles qu'elles soient et de quelque cause qu'elles proviennent, tendent à préserver les individus d'une espèce et se transmettent ordinairement à leur descendance, pourvu qu'elles soient utiles à ces individus dans leurs rapports infiniment complexes avec les autres êtres organisés et avec les conditions physiques de la vie ${ }^{15}$. [Owing to this struggle, variations, however slight and from whatever cause proceeding, if they be in any degree profitable to the individuals of a species, in their infinitely complex relations to other organic beings and to their physical conditions of life.]

Il ajoute, peu après :

La végétation à son tour aurait une grande influence sur les insectes, et l'augmentation de ceux-ci provoquerait, comme nous venons de le voir par l'exemple du Staffordshire, le développement d'oiseaux insectivores, et ainsi de suite, en cercles toujours de plus en plus complexes [in ever-increasing circles of complexity]. Ce n'est pas que, dans la nature, les rapports soient

14. Notre traduction. Nous surlignons.

15. Nous surlignons. 
toujours aussi simples que cela [Not that under nature the relations will ever be as simple as this]. La lutte dans la lutte doit toujours se reproduire avec des succès différents; cependant, dans le cours des siècles, les forces se balancent si exactement que la face de la nature reste uniforme pendant d'immenses périodes, bien qu'assurément la cause la plus insignifiante suffise pour assurer la victoire à tel ou tel être organisé. Néanmoins, notre ignorance est si profonde et notre vanité si grande, que nous nous étonnons quand nous apprenons l'extinction d'un être organisé; comme nous ne comprenons pas la cause de cette extinction, nous ne savons qu'invoquer des cataclysmes, qui viennent désoler le monde, et inventer des lois sur la durée des formes vivantes ${ }^{16}$.

Si nous laissons de côté la dernière phrase, sur l'ignorance des causes d'extinction organique et l'invocation de phénomènes inadéquats, qui résonne de façon très spinoziste, cette pensée de la relation et de ce à quoi se rapportent ou se réfèrent les individus humains fait un écho schématique prononcé à la pensée spinoziste. D’abord parce qu'il évoque la thématique des rapports biotiques et non biotiques de l'individu humain à la Nature que Spinoza appelait, d'un nom tout-à-fait général, "alimentation » ${ }^{17}$. Or cette thématique, le philosophe la liait tout à fait explicitement avec le concept de «composition individuelle» d'une part, avec le concept d'aptitude (qui est un équivalent de la complexité biologique contemporaine, terme qui ne fait pas partie du vocabulaire spinoziste) de l'autre. Une de ces occurrences les plus marquantes réside au scolie de la proposition 45 de la quatrième partie de l'Éthique:

Le Corps humain en effet est composé d'un très grand nombre de parties de nature différente qui ont continuellement besoin d'une alimentation nouvelle et variée [Corpus namque humanum ex plurimis diversae naturae partibus componitur, quae continuo novo alimento indigent et vario], pour que le Corps entier soit également apte à tout ce qui peut suivre de sa nature et que l'Âme soit également apte à comprendre à la fois plusieurs choses [ut totum Corpus ad omnia, quae ex ipsius natura sequi possunt, aeqe aptum sit, \& consequenter ut Mens etiam aeque apta sit ad plura simul intelligendum].

L'ensemble du propos spinoziste sur l'alimentation affective changeante et diverse (qu'elle soit très faiblement composée ou au contraire très composée) des parties de la composition organique d'un individu s'insère parfaitement dans le schéma conceptuel que nous avons vu plus haut. Nous pouvons le tenir pour acquis. L'intérêt de la compréhension spinoziste réside dans le fait que cette alimentation nouvelle et variée, et donc le nombre de changements accumulés par un individu, s'exprime dans des proportions infinies ou quasi-infinies. Ce qui implique, rigoureusement, que l'individu

16. Nous surlignons.

17. Pour une compréhension proche, mais différente (car liée à l'effet Baldwin) des avantages sélectifs de la plasticité individuelle liée à la complexité des interactions avec le milieu, on lira de Daniel Dennett, La conscience expliquée, Odile Jacob, Paris, I993. 
apte, pourtant fini, entretiendrait un rapport étroit avec un contexte infiniment varié. Or - c'est sur cet élément que nous voudrions terminer - ce rapport, cette référence d'un individu fini à la nature infinie trouve chez Spinoza, dans la lettre I 2 à Louis Meyer, une expression qui renforce l'expression darwinienne des "ever-increasing circles of complexity» d'une façon absolument fulgurante. Spinoza y propose une démonstration d'un type d'infini en acte, reposant sur le rapport de cercles non concentriques, selon le schéma suivant.

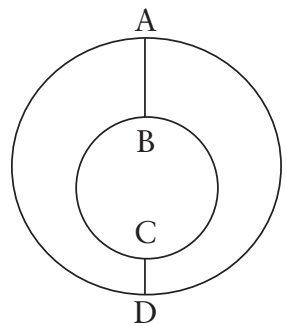

Nous ne ne revenons pas sur la démonstration spinoziste identifiant infini et impossibilité de dénombrer l'ensemble des distances séparant les deux cercles. L'intérêt réside pour nous surtout dans l'élaboration du rapport entre les deux cercles. Le cercle de diamètre $\mathrm{BC}$ se réfère d'abord, distinctement, au cercle de diamètre $\mathrm{AD}$, par un rapport simple. Il s'en distingue empiriquement par une distance de l'un à l'autre, par une différence seulement spatiale. Pourtant, après analyse (qui est en l'occurrence faite par Spinoza dans la démonstration), il est tout à fait possible de dire que le premier est en rapport avec le second d'une façon complexe, parce qu'appuyée sur une distinction infinie. Le type de rapport entre les deux cercles, au premier abord simple, est en seconde analyse tout à fait complexe. Que l'on transpose alors, et que l'on fasse de BC un individu composé, de $\mathrm{AD}$ l'individu infiniment composé de la nature, très contraignant, et l'on obtiendra une version apprêtée du modèle darwinien des cercles croissants de complexité.

\section{Conclusion}

À cette aune, que dire des rapprochements évoqués plus haut? Il est évident qu'une conceptualité de différenciation est la clef de compréhension d'un véritable héritage conceptuel de Spinoza à Darwin. Ainsi, nous ne serions pas d'accord pour dire qu'il n'existe pas une pensée de la supériorité humaine chez ces deux auteurs, pour peu que l'on comprenne l'histoire conceptuelle d'un tel terme: de la praestantia à la distinction complexe face aux circumstantiae. L'héritage conceptuel n'est donc pas seulement avéré, il est rigoureux. Enfin, il nous semble que toute investigation commençant par la piste de l'adaptation est périlleuse, dans la mesure où l'auteur anglais ne fait que récupérer une distinction transposable, mais que son véritable concept de sélection ne correspond qu'à une instance intérieure d'accommodement 
généralisé à tous les individus obligés de répondre aux exigences naturelles qui sont en nombre quasiment infinis. Certains individus, finis pourtant, sont aptes à répondre à cette exigence quasi-infinie, il est donc nécessaire de reconnaître qu'il existe chez Spinoza une forme de pensée de la complexité individuelle.

N'est-ce pas alors une sorte de vertige conceptuel: penser que Spinoza propose une piste théorique qui n'est qu'en partie suivie par Darwin, mais qui sera reprise après lui par certains biologistes ou paléo-anthropologues: l'individu apte, complexe et distinct, doit-il développer, à même sa praestantia, un équivalent mental de cette position avantageuse? Chaque individu possède une pensée en adéquation avec le type de position qu'il possède dans la nature et le type de rapport qu'il entretient avec celle-ci. Il reviendra aux pensées de l'émergence du $\mathrm{XXI}^{\mathrm{e}}$ siècle de récupérer cet aspect, en montrant que les individus complexes sont autant des unités d'intégration de changements accumulés dans leurs sous-unités de composition, que la perception unifiée du rapport de l'ensemble de ces parties, et du rapport de ce rapport à l'environnement affectif constant qui détermine l'individu ${ }^{18}$.

18. Nous pensons par exemple aux travaux de Patrick Tort, de Faustino Cordon et de Cumin Cunchillos, compilés en partie dans Faustino Cordón, «Fondements biologiques d'une théorie de la conscience", in Pour Darwin, P. Tort (dir.), et Les voies de l'émergence, Introduction à la théorie des unités de niveau d'intégration, Belin, Paris, 20I4; ainsi qu'aux travaux d'Henri Atlan, par exemple dans Les Étincelles de hasard, Seuil, Paris, 2013. 